\title{
Actitudes desfavorables hacia mujeres líderes. Un instrumento para su evaluación
}

\section{Unfavorable attitudes towards women leaders. An instrument to assess them}

\author{
María Laura Lupano Perugini ${ }^{1} \quad$ Alejandro Castro Solano \\ Consejo Nacional de Investigaciones Científicas y Técnicas, Argentina \\ Universidad de Palermo, Argentina
}

(Rec: Agosto 2011 - Acep: Octubre 2011)

\begin{abstract}
Resumen
El principal objetivo de la investigación consistió en diseñar una prueba para evaluar actitudes desfavorables hacia mujeres líderes. Sobre la técnica de Peters, Terborg y Taylor-WAMS- se diseñó el ACT-ML. Contiene 7 ítems y presenta dos dimensiones, una evalúa en que medida las personas consideran que las mujeres presentan características asociadas al liderazgo (Características Instrumentales); y la otra, el nivel de aceptación de las mujeres como líderes (Aceptación Liderazgo Femenino). Participaron 789 sujetos; 419 varones $(53,1 \%)$ y 370 mujeres $(46,9 \%)$, con promedio de 36,52 años $(D E=11,7)$. El 45,8\% $(n=361)$ de los participantes ocupa puestos de dirección, y el $54,2 \%(n=428)$ restante era subordinado. Los resultados muestran buenas propiedades psicométricas (confiabilidad y validez). Además, se analizaron diferencias significativas según sexo y tipo de puesto (líder-seguidor), hallándose diferencias a favor de los varones y seguidores que muestran mayor nivel de actitudes desfavorables hacia mujeres líderes.
\end{abstract}

Palabras clave: liderazgo, actitudes, género.

\begin{abstract}
The main objective of the investigation consisted in designing a test to evaluate unfavorable attitudes towards women leaders. Based on Peters, Terborg and Taylor test -WAMS-, it was designed the instrument ACT-ML. This one contains 7 items and presents two dimensions, one evaluates the level that people consider if women display characteristics associate with leadership (Instrumental characteristics); and the other evaluates the level of acceptance of women like leaders (Acceptance Feminine Leadership). 789 subjects participated, 419 male $(53,1 \%)$ and 370 female $(46,9 \%)$ (Age average $=36,52 ; S D=11,7) .45,8 \%(n=361)$ of the participants occupied directive position and the $54,2 \%(n=428)$ were subordinates. Results indicated good psychometric properties (reliability and validity). In addition, it was tried to verify the existence of significant differences according to sex and position (leader-subordinate), finding differences in favor of men and followers in a greater level of unfavorable attitudes to women leaders.
\end{abstract}

Key words: leadership, attitudes, gender.

\footnotetext{
Correspondencia a: María Laura Lupano Perugini. Dirección: Universidad de Palermo, Av. Dorrego 1279 (CP: 1414), Buenos Aires, Argentina. E-mail: mllupano@psi.uba.ar.
} 


\section{Introducción}

El campo constituido por el liderazgo es muy diverso e incluye un amplio espectro de teorías, definiciones, evaluaciones, descripciones y prescripciones. Por lo tanto, no existe hasta el momento una definición específica y ampliamente aceptada respecto del mismo. Sin embargo, gran parte de las escuelas que abordan su estudio, acuerdan que el liderazgo puede ser definido como un proceso natural de influencia que ocurre entre una persona -el líder-y sus seguidores. Además coinciden en que este proceso de influencia puede ser explicado a partir de determinadas características y conductas del líder, por percepciones y atribuciones por parte de los seguidores y por el contexto en el cual ocurre dicho proceso (Antonakis, Cianciolo \& Sternberg, 2004). Lord y Maher (1991) sostienen que el liderazgo es fundamentalmente un proceso atributivo resultado de un proceso de percepción social, siendo la esencia del mismo el ser percibido como líder por los otros.

Resulta importante destacar que el liderazgo constituye un fenómeno que tradicionalmente ha sido asociado al género masculino. Schein (2001) se refiere a esta particularidad sosteniendo que cuando se piensa en puestos gerenciales, se piensa en un hombre (Think manager, think male). Esto se refleja en el hecho de que una de las desigualdades más llamativas presente en la mayoría de las sociedades occidentales es la inferior ocupación, por parte de mujeres, de puestos de dirección en distintos ámbitos y organizaciones (Cuadrado, 2004). La diferencia a favor de los hombres en dicho acceso comúnmente se la ha explicado a través de la metáfora del Techo de Cristal (Federal Glass Ceiling Commission, 1995; Morrison, White \& Van Velsor, 1987). Dicha metáfora intenta mostrar que existen barreras invisibles, pero efectivas, que permiten a las mujeres avanzar solo hasta un determinado nivel en la escala jerárquica de las organizaciones.

Eagly y Carli (2007) aseguran que esta situación ha empezado a cambiar y las mujeres presentan mayor acceso, incluso en ámbitos que anteriormente eran exclusivos de los hombres. Piénsese, por ejemplo, en los cargos políticos presidenciales ocupados por mujeres en los últimos tiempos. Por esta razón, estas autoras proponen el concepto de laberinto como nueva metáfora en reemplazo de la ya mencionada. De este modo destacan que, en la actualidad, a las mujeres no les está bloqueado el acceso a los puestos más altos, pero sí deben sortear diferentes obstáculos y recorrer tortuosos caminos para poder llegar a ellos.

Ambas metáforas señalan las expectativas sociales que son depositadas en las mujeres y que perjudican su acceso a puestos de liderazgo. Se suele caracterizar a los cargos directivos con propiedades generalmente atribuidas a los hombres, como competitividad, control, autoridad u orientación hacia la tarea -rasgos instrumentales/agénticos. Estas consideraciones hacen que características propiamente femeninas como la orientación y preocupación por los otros -rasgos expresivos/ comunales - no sean consideradas esperables para los puestos de liderazgo, fomentando sesgos contra las mujeres en selección, promoción, entrenamiento y ocupación de puestos directivos (Cuadrado, 2004).

En esta línea, la Teoría de congruencia del rol hacia las mujeres líderes, propuesta por Eagly y Karau (2002), defiende que el prejuicio hacia las mujeres líderes es una de las causas de las dificultades que presentan las mujeres para acceder a puestos de liderazgo. El prejuicio procedería de la incongruencia entre las características femeninas y los requisitos del rol de líder. El constructo clave de la Teoría es el de roles de género, es decir, aquellas creencias compartidas acerca de los atributos de hombres y mujeres (Morales \& Cuadrado, 2004). Los roles incluyen dos tipos de expectativas o normas: descriptivas -expectativas compartidas sobre lo que los miembros de un grupo realmente hacen- $\mathrm{y}$ prescriptivas -expectativas compartidas sobre lo que los miembros de un grupo idealmente deberían hacer.

En términos generales, las personas tienden a creer que para ocupar y desempeñarse efectivamente en puestos de liderazgo (especialmente en las organizaciones de mayor prestigio social) es necesario desplegar cualidades masculinas, principalmente en roles definidos de modo más agéntico (e.g. roles militares). Dicha percepción ha presionado a muchas mujeres a adoptar características similares a los hombres. No obstante, cuando algunas mujeres llegan a ser consideradas igual de competentes que sus pares varones, las personas tienden a considerar que violan las normas esperadas socialmente (o prescriptivas) para las mujeres y tienden a reaccionar más negativamente frente a mujeres dominantes que frente a hombres con similares características (Butler \& Geis, 1990).

De acuerdo con lo antedicho, las mujeres líderes pueden llegar a ser evaluadas negativamente por dos razones: ya sea porque no despliegan las características que comúnmente las personas relacionan con el liderazgo efectivo o porque, en el caso de que las desplieguen, son consideradas poco femeninas. De ambas formas existe incongruencia entre el rol de liderazgo y el rol social. En consecuencia, se ven obligadas a combinar atributos asociados al estereotipo masculino (asertividad, competencia, control) con cualidades 
femeninas, con el fin de lograr no ser rechazadas principalmente por sus pares varones. En un estudio acerca de teorías implícitas acerca del liderazgo masculino y femenino (Lupano Perugini \& Castro Solano, 2008), se hallaron resultados que confirman dichas ideas. En el mencionado estudio se solicitaba a las personas que caracterizaran prototipos ideales de líderes hombres y mujeres. Se halló que a los prototipos masculinos de liderazgo se los suele relacionar con características como la capacidad de conducción y el logro de resultados obtenidos. En cambio, los prototipos femeninos eran asociados con atributos tales como la protección y la consecución de beneficios sociales, características que se corresponden con rasgos típicamente asociados al género femenino. Sin embargo, los participantes de dicho estudio consideraron que las líderes mujeres también despliegan características como la firmeza y la habilidad para superar obstáculos, las cuales son asociadas por lo general al género masculino. Dichos resultados dan cuenta de la necesidad de combinar atributos de ambos géneros para consolidar un liderazgo femenino efectivo. Los varones, en cambio, no presentan incongruencia entre ambos roles, por lo cual se ven ampliamente favorecidos para ocupar los puestos más altos en la cúspide jerárquica.

Dichas propuestas muestran la existencia de actitudes desfavorables hacia las mujeres que ocupan (o pueden llegar a ocupar) puestos de liderazgo. De acuerdo con Cortada de Kohan (2004), el estudio del constructo actitudes no es reciente (véase Eysenck, 1947; Hovlan \& Sherif, 1953; Krech \& Crutchfield, 1954; Thurstone, 1929). No existe una única definición sobre el término, se las suele entender como procesos de construcción de la realidad que guían nuestras conductas. Son concebidas como creencias, sentimientos y tendencias hacia la acción de una persona respecto a objetos, personas o ideas. Puede decirse entonces que las diferentes definiciones plantean algunas características centrales de la actitud (Ubillos, Mayordomo \& Pérez, 2003): a) es un constructo o variable no observable directamente; b) implica una organización de aspectos cognitivos, afectivos y conativos; c) tiene un papel motivacional o de impulsión de la conducta y, también, influye sobre la percepción y el pensamiento; d) es aprendida; e) es perdurable; y f) tiene un componente de evaluación o afectividad. Se considera, entonces, que una actitud tiene tres componentes: cognoscitivo, emocional y de tendencia a la acción. El cognitivo recae en las creencias acerca de personas (u objetos), especialmente en su dimensión evaluativa. El emocional refiere a los sentimientos y emociones que despiertan las personas objeto de la actitud. Por último, el componente vinculado a la acción, prepara al individuo para responder frente a las personas que despiertan las actitudes. Son aprendidas como resultado del desarrollo e interacción con objetos sociales, lo que determina que sean relativamente estables a lo largo de la vida y difíciles de modificar (Zimbardo, Ebbesen \& Maslach, 1982). En relación con esto, investigaciones previas han demostrado que las actitudes ambivalentes (positivas y negativas al mismo tiempo) son menos consistentes transituacionalmente y menos estables a lo largo del tiempo, a la vez que muestran tendencia a polarizarse (Eagly \& Chaiken, 1998).

Como se expuso previamente, las actitudes negativas refieren al fenómeno del prejuicio. La temática del prejuicio es uno de los campos más comprometidos dentro de la investigación social. Es por ello que existe un cúmulo importante de trabajos que abordan el tema, principalmente en vinculación a diferentes tipos de discriminación. Pascale (2010), en un reciente trabajo, estimó la producción con la que se cuenta hasta el momento sobre estos temas. Así, mediante la base de datos PsycINFO (Psychological Abstracts), dio cuenta que, en el período comprendido entre 1980 y 2007 , la cantidad de trabajos sobre prejuicio y discriminación es cerca de 4000 publicaciones y, lo que es mas interesante aún, se evidencia un crecimiento importante durante la última década $(10,4 \%$ de tasa de crecimiento) en comparación con el volumen total de publicaciones psicológicas (5,3\%). El término prejuicio implica la elaboración de un juicio prematuro o previo, no fundamentado en pruebas (Allport, 1954; Billig, 1986). Por lo general se reserva dicho concepto para juicios negativos referentes no tanto a una persona sino a grupos enteros. Una persona con prejuicios tiene una opinión dogmática y desfavorable hacia un determinado conjunto y, por ende, cabe esperar que se prevenga de los miembros individuales de esos grupos simplemente por considerarlos miembros de ellos (Brown, 1995; Meertens \& Pettigrew, 1997).

Se puede establecer una distinción entre el término prejuicio y discriminación: prejuicio refiere a las actitudes negativas, en cambio discriminación a comportamientos hostiles dirigidos contra los individuos objeto del prejuicio, por ejemplo la evitación o el agravio. De todos modos, Eagly (2006), reformulando las ideas originales de Kelman y colaboradores (Ver Kelman \& Pettigrew, 1959, Kelman \& Warwick, 1973) sobre la temática del prejuicio, sostiene que éste término debe ser ampliado. Para ella no debe incluir solo actitudes negativas hacia determinadas personas, sino que implica una evaluación devaluada (no necesariamente negativa) de determinados grupos cuando quieren incurrir 
en el terreno de otros. De hecho, algunos conjuntos de personas objeto del prejuicio pueden ser evaluados de manera positiva (e.g. las mujeres y sus atributos comunales) pero pasan a ser vistos de forma devaluada cuando incurren en roles no comunes a su grupo (e.g. las mujeres en rol de líderes). Siguiendo en la misma línea, algunos autores (e.g. Coenders, Scheppers, Sniderman \& Verberk, 2001; Pascale, 2010; Pettigrew $\&$ Meertens, 1995, 2001) señalan que deben diferenciarse los prejuicios obvios de otros de características más sutiles. Estos autores destacan que si bien se han realizado avances políticos y sociales significativos para lograr mayor igualdad y eliminar ciertos prejuicios desvalorizadores que, por mucho tiempo, han sido instrumentos de legitimación en diversas situaciones de dominación -sobre todo en lo relacionado a grupos étnicos o raciales-, esto no determina que los prejuicios hayan desaparecido (Antón \& Del Popolo, 2008; Banco Mundial, 2004, 2008; Bello \& Paixao, 2008).

En un contexto mundial que pregona por la igualdad, los prejuicios son mal vistos, por lo tanto no han desaparecido sino que han adoptando formas más sutiles y difíciles de identificar. Esto se hace evidente en cuanto a las nuevas formas de racismo ya que, en contextos sociales donde se promueve el anti-racismo, se practican formas indirectas de atribución de inferioridad de exogrupos. En este sentido, el nuevo racismo o cualquier otro tipo de prejuicio sutil refiere a actitudes, comportamientos y creencias encubiertas que colocan al otro en una categoría inferior (Pascale, 2010).

Por lo general se tiende a evaluar al grupo objeto de las actitudes desfavorables en comparación con otro dominante, asignando a este último todas aquellas cualidades y características que son deseadas socialmente. Estas creencias son activadas automáticamente y son resistentes al cambio, ya que las personas que las sostienen buscan continuamente información que las corrobore y desechan aquella que las contradice (Brewer, 1999).

En resumen, aplicando este concepto al grupo de las mujeres líderes, se considera que éstas son objeto de prejuicio al ser comparadas con sus pares masculinos. Los hombres son percibidos con las cualidades necesarias para convertirse en líderes, no así el grupo de las mujeres. Las actitudes desfavorables emergen, entonces, cuando éstas ocupan posiciones jerárquicas ya que son evaluadas negativamente a partir de la incongruencia percibida por parte de los seguidores entre las características típicas de su género y las requeridas para el ejercicio del liderazgo.

Se han realizado variados estudios acerca de esta temática. La mayor parte han sido efectuados utilizando el instrumento WAMS (Women as Manager Scale) de
Peters, Terborg y Taynor (1974). Dicho instrumento resulta ser el más citado en investigaciones sobre actitudes desfavorables hacia mujeres líderes (Moore, Grunberg \& Greenberg, 2003). Contiene ítems que evalúan si las personas aceptan a las mujeres como potenciales líderes así como otros tendientes a analizar si las mujeres poseen características que resulten útiles en esas posiciones. La escala fue diseñada conteniendo la mitad de ítems formulados como actitudes favorables y la otra mitad como desfavorables.

Otros estudios fueron realizados empleando la técnica ATWAM (Attitudes Towards Women As Managers) de Yost y Herbert (1985). Por ejemplo, una investigación realizada con empleados de diferentes organizaciones y estudiantes universitarios mostró que las actitudes eran más favorables si quienes respondían eran mujeres, tenían altos niveles de estudio, habían trabajado para una líder mujer y/o tenían un mayor nivel de aceptación general respecto de otras personas (Allen, Srinivas \& Sakamoto, 1997).

En la misma línea, un meta análisis realizado por Eagly, Makhijani y Klonsky (1992) mostró que las mujeres líderes eran evaluadas menos favorablemente que sus compañeros hombres. Esto sucedía principalmente cuando éstas empleaban estilos masculinos de liderazgo (directivo), cuando ocupaban roles en ámbitos dominados por hombres y cuando los evaluadores eran hombres. De acuerdo con Morales y Cuadrado (2004), el sexo del perceptor es una variable relevante a tener en cuenta en el estudio de actitudes. Los hombres tienen una concepción más masculina de liderazgo, además, debido al mayor poder social de los hombres, la tendencia de éstos a utilizar información estereotípica de género, en lugar de información sobre el desempeño individual de las mujeres en puestos jerárquicos, incrementa las posibilidades de generar mayor nivel de actitudes desfavorables por parte de los hombres (Schein, 1973, 1975; Goodwin, Operario \& Fiske, 1998). Otros estudios también reflejan la existencia de actitudes desfavorables hacia las líderes mujeres. Por ejemplo, datos presentados por la encuesta internacional de la consultora Gallup -realizada entre los años 1953 y 2000- ponen de manifiesto la preferencia a lo largo del tiempo por parte de las personas de ambos sexos hacia jefes hombres. Esta preferencia se mantuvo en las 22 naciones que Gallup encuestó (Simmons, 2001). Por otra parte, diversas investigaciones que utilizan escalas para medir actitudes hacia mujeres líderes, así como investigaciones experimentales concluyen en forma general que, si bien disminuye con el tiempo, aún existe cierto grado de desaprobación hacia mujeres líderes y 
que ésta es mayor en los hombres. No obstante, Morales y Cuadrado (2004) destacan determinadas inconsistencias en los resultados debidas, probablemente, a la variedad de medidas y muestras empeladas.

Por último, en un estudio experimental realizado por los autores (Lupano Perugini \& Castro Solano, 2010), que intentaba determinar si las características estereotípicas de las mujeres líderes y el sexo del seguidor influyen en las actitudes sostenidas por los seguidores hacia mujeres líderes, se hallaron resultados congruentes con lo expuesto ya que se demostró que se mantienen actitudes desfavorables hacia mujeres líderes, principalmente hacia aquellas que sólo presentan atributos agénticos, en cambio tienden a disminuir hacia las mujeres líderes que combinan atributos comunales y agénticos.

En función de lo previamente mencionado, el presente estudio tiene los siguientes objetivos:

1. Construir y validar un instrumento (ACT-ML) para evaluar actitudes desfavorables hacia mujeres líderes sobre la base de la técnica diseñada por Peters, Terborg y Taylor: Women As Managers Scale WAMS (1974).

2. Examinar diferencias individuales en el nivel de actitudes desfavorables hacia mujeres líderes según sexo.

3. Examinar diferencias individuales en el nivel de actitudes desfavorables hacia mujeres líderes según tipo de puesto (líder / seguidor).

Sobre la base de los antecedentes teóricos y empíricos expuestos, se formula la siguiente hipótesis:

Los hombres presentan mayor nivel de actitudes desfavorables hacia las mujeres líderes.

\section{Método}

\section{Participantes}

Participaron del estudio 789 sujetos adultos, 419 varones $(53,1 \%)$ y 370 mujeres $(46,9 \%)$ que tenían en promedio 36,52 años $(D E=11,7)$. El 45,8\% ( $n=$ 361) de los participantes ocupaba puestos de dirección o gerenciamiento, y el 54,2\% $(n=428)$ restante era subordinado. El 23,4\% $(n=185)$ trabajaba en empresas pequeñas, el $39,2 \%$ se desempeñaba en empresas medianas $(n=309)$ y el restante $36,4 \%$ en grandes empresas $(n=287)$.

De acuerdo al cargo desempeñado se definieron cinco grupos, de mayor a menor, con respecto a la categoría ocupacional. El 29,2\% era funcionario, directivo y/o profesional -se trataba de la categoría ocupacional más alta- $(n=230)$. El 22,2\% era jefe de nivel intermedio $(n=175)$. El 39,9\% $(n=315)$ trabajaba como comerciante, empleado calificado, docente, entre otros. El 6,5\% $(n=51)$ trabajaba como cuentapropista, en servicios, como chofer, empleado no calificado, entre otros. El 2,3\% restante se dedicaba a actividades no incluidas en las categorías precedentes $(n=18)$.

Con respecto al nivel educativo, la mayoría $(75,3 \%)$ estaba cursando o poseía estudios universitarios o superiores $(n=594)$. El 14,4\% $(n=114)$ afirmaba estar cursando o haber finalizado estudios terciarios; y el $10,3 \%$ restante presentaba un nivel de estudios menor -principalmente secundario completo- $(n=81)$.

La mayor parte de los participantes $(88,6 \% ; n=699)$ residía en la ciudad de Buenos Aires y el conurbano bonaerense (Argentina). El resto $(8,4 \%)$ se distribuía en provincias del interior, y solo el $0,4 \%(n=3)$ residía en el exterior. El 2,7\% $(n=21)$ no mencionó lugar de residencia.

\section{Instrumentos}

Escala de actitudes desfavorables hacia mujeres líderes 'ACT-ML' (ver cuadro 1).

Este instrumento fue construido, principalmente, sobre la base de la técnica diseñada por Peters, Terborg y Taylor-Women As Managers Scale WAMS- (1974). Se seleccionaron y adaptaron los ítems que resultaban más consistentes teóricamente y se ajustaban al contexto local.

Tiene la finalidad de evaluar la presencia de actitudes desfavorables hacia el liderazgo femenino. La presencia de actitudes desfavorables suele estar asociado con la existencia de prejuicios hacia las mujeres líderes. Dichos prejuicios suponen que las mujeres no contarían con los atributos necesarios para ejercer liderazgo.

El instrumento final consta de 7 ítems (se eliminaron dos ítems de la primera versión diseñada) que se responden de manera autoadministrable. Los sujetos deben contestar sobre la base de un formato de respuesta tipo Likert con 7 opciones que van del Total Desacuerdo al Total Acuerdo. Los ítems 2, 3, 6 y 7 se puntúan de manera inversa. El instrumento presenta dos dimensiones, una tendiente a evaluar en qué medida las personas consideran que las mujeres presentan características instrumentales comúnmente asociadas al liderazgo (Características Instrumentales); y otra, tendiente a evaluar el nivel de aceptación de las mujeres como líderes (Aceptación Liderazgo Femenino). En el apartado de Resultados se analizan las características psicométricas de la prueba. 
Cuadro 1. Escala de actitudes hacia mujeres líderes.

Instrucciones: Responda en una escala de 1 (Total desacuerdo) a 7 (Total acuerdo) a las siguientes afirmaciones acerca de las mujeres líderes.

1- Las mujeres no son lo suficientemente ambiciosas como para ser exitosas en el mundo laboral

2- Las mujeres deben tener las mismas oportunidades que los hombres de participar en programas de entrenamiento gerencial

3- Las mujeres pueden ascender en la misma medida que los hombres

4- Las mujeres no son lo suficientemente competitivas como para ser exitosas en el mundo laboral

5- Las mujeres no presentan las habilidades sociales y políticas necesarias para puestos jerárquicos

6- El trabajo realizado por mujeres líderes es igual de valorable que el realizado por hombres líderes

7- Las mujeres presentan la capacidad para adquirir las habilidades necesarias como para convertirse en líderes
Totalmente en desacuerdo $1-2-3-4-5-6-7$ Totalmente de acuerdo

Totalmente en desacuerdo $1-2-3-4-5-6-7$ Totalmente de acuerdo

Totalmente en desacuerdo $1-2-3-4-5-6-7$ Totalmente de acuerdo

Totalmente en desacuerdo $1-2-3-4-5-6-7$ Totalmente de acuerdo

Totalmente en desacuerdo $1-2-3-4-5-6-7$ Totalmente de acuerdo

Totalmente en desacuerdo $1-2-3-4-5-6-7$ Totalmente de acuerdo

Totalmente en desacuerdo $1-2-3-4-5-6-7$ Totalmente de acuerdo

\section{Procedimiento}

Los instrumentos formaban parte de una batería de pruebas más amplia que se administró con el propósito de obtener información sobre teorías implícitas, creencias y actitudes acerca del liderazgo femenino y masculino. Las tareas de recolección y entrada de datos estuvieron a cargo de un becario. El análisis de los datos se efectuó con el procesador SPSS versión 13.0.

\section{Resultados}

\section{Características psicométricas del instrumento ACT-ML}

\section{Validez Factorial}

\section{Análisis factorial exploratorio}

Teniendo como objetivo estimar la validez de la prueba, se efectuaron análisis factoriales exploratorios de primer grado utilizando el método de análisis de componentes principales con rotación tipo Varimax. No resultó necesaria la eliminación de ítems ya que éstos no presentaron peso similar en más de un factor. La solución factorial final (Test de esfericidad de Bartlet $=1704,85 p<0.01$; Índice Káiser Meyer Olkin $=0.81$ ) consta de 2 factores que explican el $53,14 \%$ de la varianza de las puntuaciones. Los factores resultantes coinciden con las dimensiones propuestas por los autores de la prueba original (Ver Tabla 1): Características Instrumentales -Factor 1-y Aceptación del liderazgo femenino -Factor 2-.

\section{Análisis factorial confirmatorio}

Seguidamente, se verificó la estructura factorial obtenida por medio del análisis factorial confirmatorio. Los parámetros del modelo original propuesto fueron estimados siguiendo el criterio de Máxima Verosimilitud. Como entrada para el análisis se utilizó la matriz de correlaciones entre los ítems de la escala. En la tabla 2 se recoge la información proporcionada por seis de los índices de ajuste más utilizados 
Tabla 1. Estructura factorial de la Escala ACT-ML $(n=789)$.

\begin{tabular}{|c|c|c|}
\hline Ítem & Factor 1 & Factor 2 \\
\hline $\begin{array}{l}\text { Las mujeres no son lo suficientemente } \\
\text { competitivas como para ser exitosas en } \\
\text { el mundo laboral (Ítem } 5 \text { ) }\end{array}$ & 0,76 & \\
\hline $\begin{array}{l}\text { Las mujeres no presentan las habilida- } \\
\text { des sociales y políticas necesarias para } \\
\text { puestos jerárquicos (Ítem } 6 \text { ) }\end{array}$ & 0,73 & \\
\hline $\begin{array}{l}\text { Las mujeres no pueden ser objetivas o } \\
\text { racionales en las situaciones laborales } \\
\text { que lo requieran (Ítem } 8 \text { ) }\end{array}$ & 0,70 & \\
\hline $\begin{array}{l}\text { Las mujeres no son lo suficientemente } \\
\text { ambiciosas como para ser exitosas en el } \\
\text { mundo laboral (Ítem 1) }\end{array}$ & 0,67 & \\
\hline $\begin{array}{l}\text { Las mujeres no pueden ser asertivas en } \\
\text { las situaciones laborales que lo requie- } \\
\text { ran (Ítem 3) }\end{array}$ & 0,66 & \\
\hline $\begin{array}{l}\text { Las mujeres presentan la capacidad para } \\
\text { adquirir las habilidades necesarias como } \\
\text { para convertirse en líderes (Ítem 9) }\end{array}$ & & 0,83 \\
\hline $\begin{array}{l}\text { Las mujeres deben tener las mismas } \\
\text { oportunidades que los hombres de par- } \\
\text { ticipar en programas de entrenamiento } \\
\text { gerencial (Ítem 2) }\end{array}$ & & 0,79 \\
\hline $\begin{array}{l}\text { Las mujeres pueden ascender en la } \\
\text { misma medida que los hombres (Ítem } 4 \text { ) }\end{array}$ & & 0,67 \\
\hline $\begin{array}{l}\text { El trabajo realizado por mujeres líderes } \\
\text { es igual de valorable que el realizado por } \\
\text { hombres líderes (Ítem } 7 \text { ) }\end{array}$ & & 0,60 \\
\hline
\end{tabular}

Tabla 2. Análisis Factorial confirmatorio del ACT- ML. Índices de ajuste para un modelo de dos factores.

\begin{tabular}{|c|c|c|c|c|c|c|c|c|}
\hline \multirow[t]{2}{*}{ ACT ML } & \multicolumn{8}{|c|}{ indices de ajuste } \\
\hline & $\chi^{2}$ & $\mathrm{p}$ & $\chi^{2} / g 1$ & GFI & AGFI & NFI & CFI & RMSEA \\
\hline $\begin{array}{l}\text { Modelo de dos factores con } \\
9 \text { ítems (original) }\end{array}$ & 383.63 & 0,00001 & 14.75 & 0.88 & 0.80 & 0.76 & 0.77 & 0.13 \\
\hline $\begin{array}{l}\text { Modelo de dos factores con } \\
7 \text { ítems (reespecificado) }\end{array}$ & 96.96 & 0,00001 & 7.45 & 0.96 & 0.92 & 0.91 & 0.92 & 0.09 \\
\hline
\end{tabular}

(García-Cueto, Gallo y Miranda, 1998): $\chi^{2} ; \chi^{2} / \mathrm{gl} ; \mathrm{GFI}$, índice de bondad de ajuste; AGFI, índice ajustado de bondad de ajuste; NFI, índice de ajuste normado; CFI, índice de ajuste comparado y RMSEA, error de aproximación cuadrático medio.
Se verificó la estructura para un modelo de dos factores -Características instrumentales y Aceptación del liderazgo femenino- (Ver Figura 1). Aunque la magnitud del estadístico $\mathrm{X}^{2}$ es elevada y significativa, lo cual indica que los datos no se ajustan al modelo propuesto, se han considerado de manera complementaria 
Figura 1. Escala de Actitudes desfavorables hacia mujeres líderes. Parámetros estimados para el modelo de 2 factores.

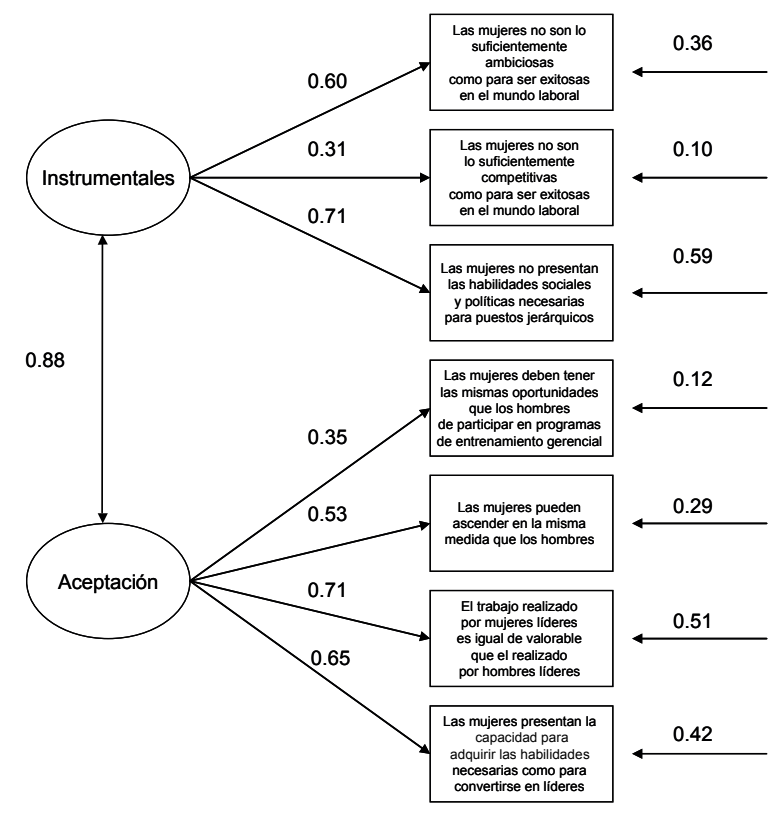

otros índices de ajuste que también se muestran en la tabla. Un análisis detallado de los resultados obtenidos en el análisis factorial confirmatorio permitió detectar errores de medida asociados a alguna de las variables observadas. Ello implicó introducir una modificación del modelo original, dando lugar a un modelo reespecificado. Dicho modelo constaba de 7 ítems de los 9 iniciales de la escala original (fueron eliminados los ítems 3 y 8 ).

\section{Fiabilidad}

Una vez que se dispuso del instrumento ACT-ML, se procedió a analizar la fiabilidad del mismo. Se calculó la fiabilidad para cada dimensión, utilizando el coeficiente alpha de Cronbach (consistencia interna). En este paso no se identificaron ítems que afectasen la fiabilidad de la prueba. Los valores obtenidos para las dimensiones de la prueba son altamente satisfactorios (Características instrumentales $=0,71$; Aceptación liderazgo femenino $=$ $0,70)$, así como para la escala total $(0,74)$.

Diferencias en el nivel de actitudes desfavorables según sexo y tipo de puesto (líder / seguidor)

A fin de responder a los objetivos 2 y 3 que se proponen verificar la existencia de diferencias estadísticamente significativas en el nivel de actitudes desfavorables según sexo y tipo de puesto (líder / seguidor), se llevó a cabo un análisis multivariado de la varianza (MANOVA) con un diseño 2 (sexo = varón/ mujer) x 2 (Puesto = líder / seguidor). Se introdujeron como variables dependientes las puntuaciones de las dos dimensiones y la puntuación total.

En cuanto a las diferencias por sexo, los resultados muestran que existen diferencias significativas entre los vectores de medias de ambos grupos $(\lambda=0.94 ; F$ $(2,784)=24,004, p<0.001, \eta=0.02)$. Siguiendo las indicaciones de Cohen (1991) con respecto al tamaño del efecto, podemos afirmar que la magnitud de esas diferencias es pequeña, limitando la significación práctica de las diferencias observadas.

Siguiendo el mismo procedimiento, se observa que existen diferencias en los vectores de medias de los grupos referentes al tipo de puesto $(\lambda=0.98 ; F(2,784)=$ $6.85, p<0.001, \eta=0.017$ ), aunque la magnitud de esas diferencias es pequeña, lo cual limita la significación práctica de las diferencias observadas.

Por último, no se encontró interacción entre sexo $\mathrm{y}$ tipo de puesto $(\lambda=0.99 ; F(2,784)=1.41, p>0.05$, $\eta=0.004)$.

Para examinar cuál dimensión del ACT-ML registra mayor variación en función de las variables independientes consideradas, se calcularon contrastes univariados. En cuanto al sexo, ambas dimensiones registran variaciones [Características Instrumentales $\mathrm{F}(1,785)=43.89, p<0,01 ;$ Aceptación del Liderazgo Femenino $(\mathrm{F}(1,785)=18.26, p<0,01)]$. Los hombres son los que registran mayor cantidad de actitudes

Tabla 3. Estadísticos descriptivos.

\begin{tabular}{|c|c|c|c|c|c|c|c|}
\hline & & \multicolumn{2}{|c|}{$\begin{array}{l}\text { Características } \\
\text { Instrumentales }\end{array}$} & \multicolumn{2}{|c|}{ Aceptación del Liderazgo Femenino } & \multicolumn{2}{|c|}{ Puntuación total } \\
\hline & & X & SD & X & SD & X & SD \\
\hline \multirow[t]{2}{*}{ Líderes } & Varones & 2.33 & 1.28 & 2.36 & 1.55 & 2.34 & 1.15 \\
\hline & Mujeres & 1.88 & 1.08 & 1.96 & 1.23 & 1.92 & 0.94 \\
\hline \multirow[t]{2}{*}{ Empleados } & Varones & 2.70 & 1.33 & 2.24 & 1.12 & 2.47 & 1.05 \\
\hline & Mujeres & 2.01 & 1.01 & 1.89 & 0.88 & 2.41 & 1.10 \\
\hline
\end{tabular}


desfavorables, consideran que las mujeres no cuentan con las características instrumentales necesarias para ser líderes y registran menor aceptación de las mismas como líderes.

En cuanto a si la persona era líder o seguidor, la dimensión del ACT-ML que más registraba variación era la de Características Instrumentales $[F(1,785)=$ $8.56, p<0,05)]$. Los subordinados son los que consideran, en mayor medida, que las mujeres no cuentan con las características instrumentales necesarias para ser líderes.

\section{Discusión}

El presente trabajo se proponía, como primer objetivo, el desarrollo de un instrumento que permitiese la evaluación de actitudes desfavorables hacia mujeres líderes. Como resultado de los análisis efectuados se ha dado lugar al ACT-ML, cuyas propiedades psicométricas lo convierten en un instrumento válido y confiable para el análisis de estos aspectos. Dicho instrumento permite evaluar en qué medida las personas consideran que las mujeres presentan características instrumentales comúnmente asociadas al liderazgo (Características Instrumentales), así como también, el nivel de aceptación de las mujeres como líderes (Aceptación Liderazgo Femenino).

Por otro lado, el análisis de los dos objetivos restantes da cuenta de resultados que son congruentes con los antecedentes teóricos y empíricos previamente expuestos.

En cuanto a las diferencias por sexo, se halló que los hombres son los que registran mayor cantidad de actitudes desfavorables, corroborando la hipótesis propuesta. Éstos consideran que las mujeres no cuentan con las características instrumentales necesarias para ser líderes y registran un menor nivel de aceptación de las mismas como líderes. Dichas diferencias eran esperables de ser encontradas ya que los hombres pertenecen al grupo dominante en el terreno del liderazgo. Tal como propone la Teoría de congruencia del rol (Eagly \& Karau, 2002) y los resultados de otros estudios organizacionales (e.g. Collinson, Knights \& Colinson, 1990; Morrison \& Von Glinow, 1990), son los hombres quienes suelen evaluar de forma más desfavorable a las mujeres líderes porque consideran que su conducta es inconsistente con muchas creencias mantenidas socialmente acerca de la conducta femenina deseable. Además las suelen ver como nuevas competidoras, aparte de sus colegas masculinos, con intención de ganar poder y autoridad en las organizaciones (Eagly \& Carli, 2007).
En el caso de las diferencias según tipo de puesto (líder/seguidor), se halló que los subordinados son los que consideran, en mayor medida, que las mujeres no cuentan con las características instrumentales necesarias para ser líderes. Si se considera que aún sigue habiendo disparidad en cuanto a la tasa de cargos directivos ocupados por hombres respecto de las mujeres, entonces, es dable pensar que muchos de los sujetos que respondieron no tengan demasiada experiencia en ser liderados por mujeres. Resultados previos (véase Allen, Srinivas \& Sakamoto, 1997) muestran que el haber tenido líderes mujeres aumenta el nivel de actitudes favorables hacia ellas.

Futuras investigaciones deberán analizar con mayor detalle cuestiones referentes a la conformación de los planteles de las organizaciones, teniendo en cuenta el número de empleados hombres y mujeres y el sexo de sus líderes. Esto resulta relevante ya que algunas investigaciones muestran que el contexto organizacional puede ser uno de los factores que dificulte el acceso de las mujeres a los puestos de dirección. Según Cuadrado Guirado, Navas Luque y Molero Alonso (2007), algunas organizaciones siguen promoviendo estilos masculinos de liderazgo. De esta manera, la ocupación de puestos jerárquicos queda relegada para las organizaciones dedicadas a actividades tradicionalmente femeninas y/o aquellos contextos en los que haya un predomino de mujeres en puestos directivos (e.g. instituciones educativas). Lo antedicho resalta la necesidad de evaluar las actitudes hacia mujeres líderes según los diferentes contextos en los que pueden desempeñarse.

Si bien en algunos ámbitos se siguen promoviendo estilos masculinos de liderazgo, ciertos cambios actuales en las organizaciones benefician a las mujeres, como por ejemplo la preferencia por estilos más transformacionales o democráticos de liderazgo que suelen ser mayormente adoptados por ellas. De esta forma Eagly y Carli (2004) suponen que la mencionada incongruencia de roles se vería minimizada ya que las organizaciones demandan de sus líderes cada vez más características agénticas pero también comunales (Cuadrado Guirado, Navas Luque \& Molero Alonso, 2007). Futuras investigaciones deberán tener en cuenta estos cambios y analizar si producen una reducción en los niveles de actitudes y evaluaciones desfavorables hacia mujeres líderes.

En adición a lo mencionado en el último párrafo, debe destacarse que también es necesario promover investigaciones que aborden las mismas dificultades que se les presentan a las mujeres en el campo del 
liderazgo pero en relación con otras minorías que también pueden verse perjudicadas. En relación con esto antedicho, Chin (2010) destaca la necesidad de ampliar las actuales teorías de liderazgo a fin de incluir cuestiones atinentes a equidad, diversidad y justicia social, teniendo en cuenta el actual contexto internacional que incluye una creciente globalización con consecuentes cambios sociales y demográficos. Por lo tanto, resulta relevante la incorporación de referencias a temas culturales en las investigaciones sobre liderazgo. Según la autora mencionada, en un relevo de publicaciones sobre liderazgo, de un total de 2207 referencias, solo 200 incorporaban algún tópico cultural. Esto da cuenta de que se tendió a dar una mirada etnocéntrica sobre el tema (Zweigenhaft \& Domhoff, 2006). El mundo del trabajo de hoy, además de ser sumamente complejo, está caracterizado por su dimensión global. Este fenómeno consiste en la ausencia de barreras y límites para ejercer el comercio, el alto impacto de las nuevas tecnologías de la información, el traslado de empresas a otros países y la consecuente migración de personal directivo y subalterno tanto de forma permanente como transitoria (Thomas, 2008). Lo anterior da cuenta de que comienza a delinearse un escenario distinto en materia de liderazgo donde es frecuente encontrar, no solo mujeres disputando los cargos más altos, sino que se evidencia, cada vez con mayor frecuencia, cómo personas de otras nacionalidades, etnias, ascendencias culturales y religiosas pueden ocupar puestos directivos en países distintos al de origen (Sánchez-Hucles \& Davis, 2010).

\section{Referencias}

Allen R.W, Srinivas S. \& Sakamoto, S. (1997). Making the room at the top: chipping the glass ceiling. Management \& Human Resources, 123-134.

Allport, G.W. (1954). The nature of prejudice. Reading, M.A: Addison-Wesley.

Antón J. \& Del Popolo F. (2008). Visibilidad estadística de la población afrodescendiente de América Latina: aspectos conceptuales y metodológicos. Santiago de Chile: CEPAL / SEGIB.

Antonakis J., Cianciolo A., \& Sternberg R. (2004). The nature of leadership. Thousand Oaks: Sage Publications.

Banco Mundial (2004). Desigualdad en América Latina y el Caribe ¿Ruptura con la historia? Washington, DC: WB.

Banco Mundial (2008). Report: Outsiders? The changing patterns of exclusion in Latin America and the Caribean. Washington, DC: WB.

Bello A. \& Paixao A. (2008). Estado actual del cumplimiento de los derechos civiles, políticos, económicos, sociales y culturales de la población afrodescendiente en América Latina. Santiago de Chile: CEPAL.

Billig, M. (1986). Racismo, prejuicio y discriminación. En S. Moscovici (Ed.), Psicología Social (pp. 575- 600). Barcelona: Paidós.

Brewer, M.B. (1999). The psychology of prejudice: Ingroup love or outgroup hate? Journal of Social Issues, 55, 429-444.
Brown, R. (1995). Prejudice: Its social psychology. Cambridge, MA: Blackwell.

Butler, D \& Geis, F. L. (1990). Nonverbal affect responses to male and female leaders: Implications for leadership evaluations. Journal of Personality and Social Psychology, 58, 48-59.

Chin J.L. (2010). Introduction to the Special Issue on Diversity and Leadership. American Psychologist, 65 (3), 150-156.

Coenders M., Scheppers P., Sniderman P.M. \& Verberk G. (2001). Blatant and subtle prejudice: dimensions, determinants and consequences. European Journal of Social Psychology, 31, 281- 297.

Cortada de Kohan, N. (2004). Teoría y Métodos para la construcción de Escalas de Actitudes. Buenos Aires: Lugar.

Cuadrado Guirado, I. Navas Luque, M. \& Molero Alonso, F. (2006). Mujeres y Liderazgo. Claves Psicosociales del Techo de Cristal. Madrid: Sanz y Torres.

Cuadrado, I. (2004). Valores y rasgos estereotípicos de género de mujeres líderes. Psicothema, 16 (2), 270- 275.

Eagly A.H. \& Carli L.L. (2007). Through the labyrinth. The truth about how women become leaders. Boston: Harvard Business School Press.

Eagly A.H., Makhijani, M-G. \& Klonsky, B.G. (1992). Gender and the evaluation of leaders: A meta-analysis. Psychological Bulletin, 111, 3-22.

Eagly, A. H. \& Karau, S. J. (2002). Role congruity theory of prejudice toward female leaders. Psychological Review, 109, 573- 598.

Eagly, A. H. \& Carli L. (2004). Women and Men as Leaders. En Antonakis J., Cianciolo A. \& Sternberg R. (2004). The nature of leadership. (pp. 279- 301). Thousand Oaks: Sage Publications.

Eagly, A. H. \& Chaiken, S. (1998). The psychology of attitudes. Fort Worth, TX: Harcourt Brace Jovanovich.

Eagly, A. H. \& Carli L. (2004). Women and Men as Leaders. En Antonakis J., Cianciolo A. \& Sternberg R. (2004). The nature of leadership. (pp. 279- 301). Thousand Oaks: Sage Publications.

Eagly, A.H. (2006). Prejudice: Toward a more inclusive understanding. En A.H. Eagly, R.M. Baron \& V.L. Hamilton (Eds.), The Social Psychology of Group Identy and Social Conflict. Theory, Application and Practice. Washington: APA.

Eysenck, H.J. (1947). Primary social attitudes. Int. J. Opinion and Attitudes Vol 1.

Federal Glass Ceiling Commission. (1995). Good for business: Making full use of the nation's human capital: The environment scan: A fact finding report of the Federal Glass Ceiling Commission. Washington, DC: U.S. Government Printing Office.

Hovlan, C.I. \& Sherif, M. (1953). Communication and persuasion. New Heaven: Wiley.

Kelman H.C. \& Pettigrew, T.F. (1959). How to understand prejudice. Commentary, 28, 436-441.

Kelman, H.C. \& Warwick, D.P. (1973). Bridging micro and macro approaches to social change: A social-psychological perspective. In G. Zaltman (Ed.). Processes and phenomena of social change (pp. 13-59). New York. Wiley.

Kelman, H.C. \& Warwick, D.P. (1973). Bridging micro and macro approaches to social change: A social-psychological perspective. In G. Zaltman (Ed.). Processes and phenomena of social change (pp. 13-59). New York. Wiley.

Krech D. \& Crutchfield, R.S. (1954). Individual in society. New York: McGraw Hill

Lord, R. \& Maher K. (1991). Leadership and information processing. London: Routledge.

Lupano Perugini, M.L. \& Castro Solano, A. (2010). Estereotipos de género, sexo del líder y del seguidor: su influencia en las actitudes hacia mujeres líderes. Estudios de Psicología. Manuscrito enviado para su evaluación.

Lupano Perugini, M.L. \& Castro Solano A. (2008). Liderazgo y género. Identificación de prototipos de liderazgo efectivo. Perspectivas en Psicología. Revista de Psicología y Ciencias Afines, 5(1), 69-77. 
Meertens, R.W. \& Pettigrew, T.F. (1997). Is subtle prejudice really prejudice? Public Opinion Quarterly, 61, 54-71.

Moore, S., Grunberg, L. \& Greenberg, E.S. (2003). Development and validation of the stereotype beliefs about women managers Scale. Workplace change, 1-26.

Morales, J. F. \& Cuadrado, I. (2004). Introducción: Teoría de congruencia de rol del prejuicio hacia líderes femeninos. Rev. de Psicol. Gral. y Aplic., 57 (2), 135- 146.

Morrison A.M. \& Von Glinow, M.A. (1990). Women and minorities in management. American Psychologist, 45, 200-208.

Morrison, A. M., White, R.P, \& Van Velsor, E. (1987). Breaking the glass ceiling: Can women reach the top of America's largest corporations? Reading, MA: Addison- Wesley.

Pascale, P. (2010). Nuevas formas de racismo: estado de la cuestión en la psicología social del prejuicio. Ciencias Psicológicas, 4 (1), 57-70.

Peters, L. H., Terborg, J. R., \& Taynor, J. (1974). Women as managers scale: A measure of attitudes toward women in management positions. JSAS Catalog of Selected Documents in Psychology, 4, 27.

Pettigrew, T.F. \& Meertens R.W. (2001). In defense of the subtle and blatant prejudice concept: A retort. European Journal of Social Psychology, 31, 299-309.

Pettigrew, T.F., \& Meertens, R.W. (1995). Subtle ad blatant prejudice in western Europe. European Journal of Social Psychology, 25, 57-75.

Sanchez-Hucles, J. V., \& Davis, D. D. (2010). Women and women of color in leadership: Complexity, identity, and ntersectionality. American Psychologist, 65, 171-181.

Schein, V. E. (1973). The relationship between sex role stereotype and requisite management characteristic. Journal of Applied Psychology, 57, 95-100.
Schein, V. E. (1975). The relationship between sex role stereotype and requisite management characteristic among female managers. Journal of Applied Psychology, 60, 340-344.

Schein, V. E. (2001). A global look at psychological barriers to women's progress in management. Journal of Social Issues, 57, 675- 688.

Simmons, W. W. (2001). When it comes to choosing a boss, American still prefer men. Recuperado el 15 de noviembre de 2010, de http:// www.gallup.com/poll/releases/pr010111.asp.

Thomas, D. (2008). Cross-cultural management. Essential Concepts. Londres; Sage.

Thurstone, L.L. (1929). Theory of attitude measurement. Psychological Review, 36, 222-241.

Ubillos, S., Mayordomo, S. \& Pérez, D. (2003). Actitudes: definición y medición. Componentes de la actitud. Modelo de la acción razonada y acción planificada. En D. Páez, I. Fernández, S. Ubillos \& E. Zubieta (Eds.), Psicología social, cultura y educación. Madrid: Pearson.

Yost, E. \& Herbert, T. (1985). Attitudes toward women as managers (ATWAM) scale. San Diego: Pfeiffer / Jossey Bass.

Zimbardo, P., Ebbesen, E. \& Maslach, C. (1982). Influencia sobre las actitudes y modificación de conducta. Bogotá: Fondo Educativo Interamericano.

Zweigenhaft, R. L. \& Domhoff, G. W. (2006). Diversity in the power elite: How it happened, why it matters. Lanham, MD: Rowman \& Littlefield. 